\title{
Financial information processing
}

\author{
Shuo BAI ${ }^{1, \dagger}$, Shouyang WANG ${ }^{2}$, Lean $\mathrm{YU}^{2}$, Aoying ZHOU ${ }^{3}$ \\ 1 Shanghai Stock Exchange, Shanghai 200120, China \\ 2 Academy of Mathematics and Systems Science, Chinese Academy of Sciences, Beijing 100190, China \\ 3 Software Engineering Institute, East China Normal University, Shanghai 200062, China
}

(c) Higher Education Press and Springer-Verlag 2009

The rapid growth in financial data volume has made financial information processing more and more difficult due to the increase in complexity, which has forced businesses and academics alike to turn to sophisticated information processing technologies for better solutions. A typical feature is that high-performance computers and advanced computational techniques play ever-increasingly important roles for business and industries to have competitive advantages. Accordingly, financial information processing has emerged as a new cross-disciplinary field integrating computer science, mathematics, financial economics, intelligent techniques, and computer simulations to make different decisions based on processed financial information.

Usually, the complexities involved in financial information processing grow at least as fast as, if not faster than, the tools and methodologies available to those actually responsible for processing financial information in real life situations as well as academic researchers, implying that financial information processing continues to be an under-researched field. Having agreed on this basic fact, the guest editors determined that the purpose of this special section should be "to assess the current state of knowledge about financial information processing, and to generate and throw open for discussion more ideas, hypotheses, and theories, the specific objective being to determine the directions for further research."

This special section presents some new progresses in financial information processing that are expected to trigger thoughts and deepen further research. For this purpose, nine papers were selected from 12 invited talks and 22 submissions related to financial information processing from different countries and regions. The authors of the selected papers are from France, Australia, Hong Kong, the Mainland of China, Japan, and the USA, respectively. In addition, all the selected papers went through a standard review process of the journal, and the authors made the necessary revision in view of the reviewing comments. The selected papers include "Risk aversion and agents' survivability in a financial market" by Serge Hayward; "Stock prediction: an event-driven approach based on bursty keywords" by Di Wu, Gabriel Pui Cheong Fung, Jeffrey Xu Yu, and Qi Pan; "Forecasting foreign exchange rates with an improved BP learning algorithm with adaptive smoothing momentum terms" by Lean Yu, Shouyang Wang, and Kin Keung Lai; "Daily prediction of short-term trends of crude oil prices using neural networks: exploiting multimarket dynamics" by Heping Pan, Imad Haidar, and Siddhivinayak Kulkarni; "A class of classification and regression methods by multi-objective programming" by Dongling Zhang, Yong Shi, Yingjie Tian, and Meihong Zhu; "Benchmarking binary classification models on data sets with different degree of imbalance" by Ligang Zhou and Kin Keung Lai; "A novel PPGA-based clustering analysis method for business cycle indicator selection" by Dabin Zhang, Lean Yu, Shouyang Wang, and Yingwen Song; "Macro finance early warning system" by Guihuan Zheng, Xun Zhang, Wei Shang, and Shanying Xu; and "Algorithmic trading system: design and applications" by Feng Wang, Keren Dong, and Xiaotie Deng. The guest editors hope that the papers published in this special section will be of value to academic researchers and business practitioners, and will provide a clearer sense of direction for further research as well as facilitate the use of existing methodologies in a more productive manner.

${ }^{\dagger}$ Profs. Shuo BAI, Shouyang WANG, Lean YU and Aoying ZHOU are the guest editors of the special section on Financial Information Processing.

E-mail: sywang@amss.ac.cn, yulean@amss.ac.cn 
The guest editors would like to place on record their sincere thanks to Prof. Ruqian Lu, the Executive Editor-inChief of Frontiers of Computer Science in China, for this very special opportunity provided to us to contribute to this special section. The guest editors have to thank all the referees for their kind support and help. Finally, the guest editors would like to thank the authors of all the submissions to this special section for their contributions. Without the support of the authors and the referees, it would have been impossible to make this special section for our readers. It is hoped that readers can find some topics of interest and benefit to them. The guest editors also hope that this special section will inspire researchers in the fields of financial information processing to explore more creative contributions in their research fields. 